\title{
A Correlative Bimodal Surface Imaging Method to Assess Hyphae-Rock Interactions.
}

Odeta Qafoku $^{1 *}$, Rebecca A. Lybrand², Vaithiyalingam Shutthanandan ${ }^{1}$, Rachel E. Gallery ${ }^{3}$, Jason C. Austin ${ }^{4}$, Paul A. Schroeder ${ }^{4}$, Jennifer Fedenko ${ }^{2}$, Erin Rooney ${ }^{2}$, Dragos G. Zaharescu ${ }^{5}$

${ }^{1}$ Pacific Northwest National Laboratory, W. R. Wiley Environmental Molecular Sciences

Laboratory, Richland, WA, USA.

${ }^{2}$ Oregon State University, Corvallis, OR, USA.

${ }^{3}$ University of Arizona. Tucson, AZ, 85721, USA.

${ }^{4}$ University of Georgia, Athens, GA 30602, USA.

${ }^{5 .}$ Georgia Institute of Technology, Atlanta, GA, USA.

*Corresponding Author: Odeta.Qafoku@pnnl.gov

Mineral weathering at the grain-microbe interface is driven by biological processes that include biophysical degradation and biochemical dissolution of mineral particles [1-3]. Microorganisms actively support terrestrial ecosystems by extracting and transferring rock-derived nutrients via direct and indirect biological weathering mechanisms. However, fundamental knowledge gaps exist in characterizing biogeochemical processes that transform microbe-mineral interfaces at submicron scales, particularly in natural settings (i.e., in Earth's critical zone, from the tree tops to the bedrock where meteoric waters have influence).

The objective of this research is to use a correlative bimodal high-resolution microscopy technique, helium ion microscopy (HIM) in conjunction with scanning electron microscopy equipped with energy-dispersive X-ray spectroscopy (SEM/EDS), to detect and assess potential biogeochemical drivers of mineral weathering in natural settings. Nylon mesh bags of different sizes (coarse - 35 $\mu \mathrm{m}$, and fine $-0.5 \mu \mathrm{m})$ were filled with pristine basalt, granite, and quartz grains $(53-250 \mu \mathrm{m})$ and deployed in surface soils $(0-10 \mathrm{~cm})$ of contrasting ecosystems (semiarid to humid) for up to three years. The mesh bags were designed to include $(35 \mu \mathrm{m})$ and exclude $(0.5 \mu \mathrm{m})$ direct fungal hyphae and microbial contact with mineral surfaces. Both mesh sizes allow for solute and nanosize solid interactions with deployed mineral grains.

A reference locator grid method was applied to analyze individual grains exposed to the natural environment using: a) HIM to capture micron to sub-nanometer scale mineral-organic interactions and; b) SEM/EDS to reveal chemical distribution and create elemental maps at same locations. The bimodal imaging method allowed for i) identifying the intricate morphology of exposed mineral surfaces prior to and following exposure to biogeochemical processes in the field and ii) assessing grain composition at the same referenced interface.

We visualized individual mineral grains and associated mineral-organic matter interactions at the micro- to nanoscale with HIM and detected selective locations of biomechanical weathering, secondary mineral precipitation, biofilm formation, and grain coatings across the contrasting climates [4]. The microscopy results enhanced our understanding of incipient biomechanical mineral weathering in complex field systems through identifying the expansion of mineral sheets by soil fungi at sub-nanometer resolution. Likewise, the microscopy data provided evidence of the early bio-mineralization processes occurring in the field humid environments through identification of secondary crystallites and/or formation of surface coating on mineral grains. 
Coupling the superior imaging capabilities of HIM with the chemical analysis by SEM/EDS helped elucidate the strong association between hyphae growth and composition of mineral grains in natural settings (Figure 1), and helped characterize the Fe-rich nature of nano-crystallites and/or surface coatings on grains after deployment. Overall, this study illustrates how a combination of HIM and SEM/EDS can be effectively applied in Earth and ecosystem sciences to elucidate the complex nanoscale environment of microbe-rock interfaces and assess the biologically driven processes in field settings, which are keystone in understanding incipient biogeochemical cycles.

\section{References}

[1] J. F. Banfield et al, Proc. Natl. Acad. Sci. 96, (1999), 3404-3411.

[2] D. G. Zaharescu et al, Scientific Reports, 7 (2017), 43208.

[3] R. Lybrand et al, Chem. Geol. 381, (2014) 26-39.

[4] R. Lybrand et al, Scientific Reports, in Review.

[5] A portion of the research was performed using EMSL (grid.436923.9), a DOE Office of Science User Facility sponsored by the Office of Biological and Environmental Research.

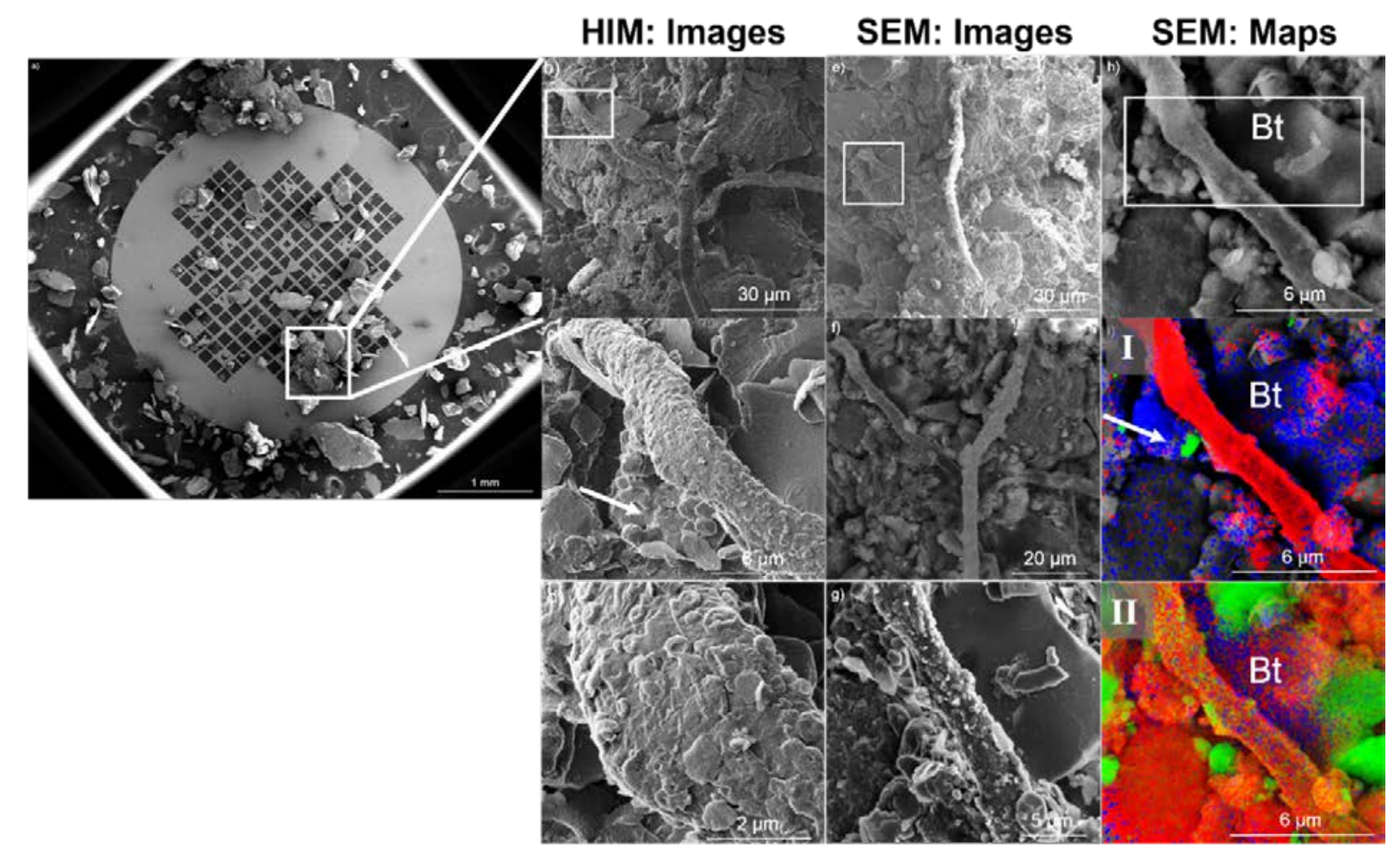

Figure 1. Demonstration of bimodal HIM-SEM approach using a granite substrate sample from the humid pine forest environment (Calhoun Critical Zone Observatory). The left image shows mineral grains, deployed in 0-10 cm surface soil for one year, scattered over a reference locator grid. The collection of images on the right show mineral-hyphae interfaces imaged at high resolution, nanoscale with HIM and submicron scale with SEM. EDS elemental maps of same microbial-rock interfaces (color images) reveal hyphae associated with biotite grain (Bt) and location of iron-rich aggregates. Chemical distribution maps were generated for I) $\mathrm{C}$ (red), Fe (blue), and Ti (green) and for II) Si (green), Al (red), and K (blue). 